\title{
Psychological Repercussions of Morbid Adherent Placenta (MAP) Patients
}

\author{
Laila Yahya A. Alhubaishi \\ Department of Obstetrics \& Gynecology, Latifa Hospital, Dubai Health Authority, Duabi, UAE \\ Email: atiffazari@hotmail.co.uk
}

How to cite this paper: Alhubaishi, L.Y.A. (2019) Psychological Repercussions of Morbid Adherent Placenta (MAP) Patients. Open Journal of Obstetrics and Gynecology, 9, 1284-1289.

https://doi.org/10.4236/ojog.2019.99124

Received: August 20, 2019

Accepted: September 22, 2019

Published: September 25, 2019

Copyright ( 2019 by author(s) and Scientific Research Publishing Inc. This work is licensed under the Creative Commons Attribution International License (CC BY 4.0).

http://creativecommons.org/licenses/by/4.0/

\section{(c) (i) Open Access}

\begin{abstract}
Morbidly adherent placenta (MAP) is a major cause of maternal morbidity and cause of severe fear and stress to the patient, family and the treating staff. Proper counseling and sympathetic case handling and management is of great relief. Detailed explanation for the pathology and the treatment plan will support the psychology of the patient and surrounding.
\end{abstract}

\section{Keywords}

Morbid Adherent Placenta, Maternal Morbidity, Maternal Mortality, Psychological Repercussions

\section{Introduction}

Placenta accreta is defined as abnormal trophoblast invasion of part or all of the placenta into the myometrium of the uterine wall [1].

Placenta accreta spectrum, formerly known as morbidly adherent placenta, refers to the range of pathologic adherence of the placenta, including placenta increta, placenta percreta, and placenta accreta.

The increasing rate of placenta accreta over the past four decades is likely due to a change in risk factors, most notably the increased rate of cesarean delivery.

Placenta accreta spectrum is considered a high-risk condition with serious associated morbidities; therefore, The American College of Obstetricians and Gynecologists (ACOG) and the Society for Maternal-Fetal Medicine (SMFM) recommend these patients receive level III (subspecialty) or higher care.

Study of the association between placenta Accreta and its psychological effect on patients has always been an interesting area of research. Increasing attention to psychological well-being has led to an interest in less researched aspect of the association between obstetric complications and chronic psycho- 
logical problems. It counseled about the diagnosis and potential sequel (e.g. hemorrhage, blood transfusion, cesarean hysterectomy, maternal intensive care unit admission).

Consultation with a maternal-fetal medicine specialist is desirable, and transfer to a Center of Excellence for placenta accreta is strongly advised [2].

Planned delivery at a center experienced with this condition is recommended whenever possible. Ideally, preoperative coordination with anesthesiology, maternal-fetal medicine, neonatology, and expert pelvic surgeons (very often gynecologic oncology or female pelvic medicine and reconstructive surgeons) can assist in proper preparations and allow the woman to ask questions, be counseled about the high likelihood and need for cesarean delivery or hysterectomy and potential complications, discuss anesthetic planning, and prepare for delivery. The use of a consistent multidisciplinary team improves maternal outcomes and can drive internal continuous quality improvement as progressive experience is gained by that same group [3] [4] as well as, consistent access to interdisciplinary staff with expertise in critical care (i.e., critical care subspecialists, hematologists, cardiologists, and neonatologists).

Unfortunately, the practice of medicine requires the occasional communication of bad news. Within obstetric and gynecology, the bad news may be lifethreatening (Organs injuries, PPH, Blood transfusion, Hysterectomy, Long stay in the Hospital, financial reasons. Away from the Family and Kids and fear of death), each type of news clearly differs in severity, but to the concerned, nervous, anxious patient, each represents bad news that may negatively impact their future. In other cases, the bad news that physicians must communicate is a result of an iatrogenic error or surgical complication, where the unforeseen event was due to human error.

Such surgical complications are very challenging for physicians to discuss, as they may feel embarrassed and culpable for such errors and may even fear future litigation. The Accreditation Council for Graduate Medical Education (ACGME), the governing body responsible for accrediting the majority of graduate medical training programs, considers "Interpersonal skills and communication" to be among the six core competencies for completion of residency. Interestingly, the delivery of bad news is not explicitly included as a key communication skill to be taught during graduate medical training. It is reasonable, therefore, to assume that many resident physicians complete training without formal education in the communication of bad news.

Bad news is broken by hospital specialists, and this is what most patients expect and desire [5] [6]. The specialist was usually a surgeon.

As Placenta accreta is one of the major obstetric complications with burdensome treatment procedures, patients are likely to have psychological health problems beyond somatic complications. Disease burden increases because of repeated visits to the hospital, repeated laboratory tests and frequent and close monitoring of symptoms in detecting emergency complications. 


\section{Overview and Background}

Placenta accreta is defined as abnormal invasion of trophoblasts of part or all of the placenta into the myometrium. Rates of placenta accreta are increasing; studies from the 1970s-1980s had shown the prevalence of placenta accreta was between 1 in 2510 and 1 in 4017 in comparison with a rate of 1 in 533 from 1982-2002. [6]. Therefore, the psychological impact of placenta accreta has to be further elucidated.

Obstetricians manage patients with placenta accreta in outpatient clinics, ante-natal wards and operation theatre with an endeavor in order to provide best maternal and fetal outcome. At the same time, it is equally crucial to give adequate attention to the psychological disturbances associated with placenta accreta. Bearing in mind the predisposing factors for adverse psychological reaction helps obstetricians to minimize and overcome these problems. Various causes of psychological trauma in patients with placenta accreta have been highlighted. For instance, majority of patients diagnosed with placenta accreta have heard about this obstetric complication for the first time from their obstetrician at the time of the diagnosis. Majority of patients react to this stressor by various defense mechanisms like denial, fear, anxiety or even more severe reactions like post-traumatic stress disorder or depression.

Placenta accreta is considered a psychological stressor not only for the patient, but also for her spouse and children. This is explained by the necessity for admission to the hospital in the last months or weeks of pregnancy for close observation which separates the patient from her children, increases financial burden and triggers more adverse psychological outcomes. Another predisposing factor is that caesarian section for placenta accreta carries a high risk for intra-operative complications that puts the patient in a state of anxiety and fear of death. Possibility for emergency hysterectomy on the contrary makes the patient perturbed about her fertility and drives her to seek alternative management modalities to preserve her uterus. Psychological measures should be performed to minimize these problems in patients with placenta accreta. These include adequate explanations, careful reassurance and to provide familiar surroundings of medical and nursing staff starting from the early time of diagnosis.

We aimed to examine the long-term psychological adjustment of patients in the terminal phase of their illness and to compare this with patient perceptions of their doctors' attitudes and skills, including their experiences of receiving bad news.

\section{Diagnosis and Ante-Natal Care}

The role of obstetricians is to provide individualized and evidence-based advices to patients with placenta accreta. Patient should be given the opportunity to develop a relationship with the healthcare team, which builds trust, as well as emotional and psychological support. This allows the patient to coordinate time away from family and work and to consult with multidisciplinary team special- 
ists. Obstetricians should have full discussion of the disease itself and to provide ample time to allow the patient and her family to ask and receive elucidated answers to their inquiries. Use of antenatal corticosteroids for lung maturation is appropriate in women with antenatally diagnosed accreta and anticipated delivery before $370 / 7$ weeks of gestation and is consistent with current gestational age-based recommendations [4].

Discussing Surgical Complications, despite the obvious similarity between delivering bad news and discussing surgical complications, there is For many physicians, these discussions are perhaps more anxiety-provoking and difficult than any other bad news that can be shared, as physicians may feel they are to blame for surgical complications, and may speak cautiously for fear of potential malpractice litigation. several studies have found that physicians involved in serious adverse events suffer profound emotional effects, including fear, guilt, anger, embarrassment, humiliation, anxiety, depression, and post-traumatic stress disorder as same as patients.

Patient should be given thorough information about placenta accreta and subsequent antenatal, natal and postnatal plan of care.

During pregnancy, patient needs to attend more frequent antenatal visits and undergo close monitoring under specialized multidisciplinary team. In vast majority of cases, patient has to be admitted to the hospital in the last weeks of pregnancy for close observation till the planned day of delivery; which has both financial and social burden on uninsured patients and those having children, respectively. Thus, these important information should be disclosed to the patient from early time of diagnosis. Further discussion should be taken in regards to mode and time of delivery, intraoperative complications such as post-partum hemorrhage (PPH) and the possibility for blood transfusion, which could be a substantial issue to disclose to patients with certain religious beliefs.

Further history should be taken regarding patient's wishes for further pregnancies and to discuss different modalities for management of placenta accreta accordingly. She should be aware about possible intra-operative emergency hysterectomy and a written consent form should be taken and signed by both, patient and her spouse. Psychological support should be carefully given for this point as this is considered a sentimental issue for majority of patients even those who does not have plans for further pregnancies.

\section{Delivery}

Cesarean section is the only modality of choice for delivery in patients with placenta accreta. This adds more to the adverse psychological outcomes to the patient as it aggravates her feelings of fear, anxiety and disquietudes bearing in mind all complications of cesarean section including $\mathrm{PPH}$, emergency hysterectomy and possible death.

For this reason, patient should be counseled about different modalities for controlling intra-operative complication e.g. uterine artery embolization or liga- 
tion for PPH. However, it was recently suggested that a severe $\mathrm{PPH}$ managed by uterine artery embolization might result in major psychological impact for the patient and her spouse, in both short- and long-term. A study was done in 2010 to estimate the long-term psychological effect of severe PPH in women whose uterus was preserved after PPH. Psychological management was proposed in only $66 \%$ of the cases, although negative repercussions were observed in $41 \%$ [7].

\section{Post Delivery}

Patients with placenta accreta usually require longer postpartum hospital stay than other females. This longer stay in the hospital is needed for patients to recover from physical and psychological trauma caused by the surgery.

Additional surgical interventions during caesarean section for those patients e.g. ureteral stent, makes post-operative recovery period somehow longer. This consequently puts more financial burden on the patient especially in those lacking medical insurance.

To learn that one has a life-threatening disease is a major event, and many patients recalled it with clarity even years later. The event itself did not seem to cause long-term psychological morbidity, although the findings must be interpreted with caution.

\section{Conclusion}

Women's satisfaction about the management used in placenta accreta and its psychological impact have not been fully studied and evaluated. It is of interest to conduct further studies in order to address the long term psychological effect of obstetric complications.

\section{Conflicts of Interest}

The author declares no conflicts of interest regarding the publication of this paper.

\section{References}

[1] Usta, I.M., Hobeika, E.M., Musa, A.A., Gabriel, G.E. and Nassar, A.H. (2005) Placenta Placenta Accreta: Risk Factors and Complications. American Journal of $O b$ stetrics \& Gynecology, 193, 1045-1049. https://doi.org/10.1016/j.ajog.2005.06.037

[2] Silver, R.M., Fox, K.A., Barton, J.R., et al. (2015) Center of Excellence for Placenta Accreta. American Journal of Obstetrics \& Gynecology, 212, 561. https://doi.org/10.1016/j.ajog.2014.11.018

[3] Shamshirsaz, A.A., Fox, K.A., Salmanian, B., Diaz-Arrastia, C.R., Lee, W., Baker, B.W., et al. (2015) Maternal Morbidity in Patients with Morbidly Adherent Placenta Treated with and without a Standardized Multidisciplinary Approach. American Journal of Obstetrics \& Gynecology, 212, 218.e1-9.

[4] Shamshirsaz, A.A., Fox, K.A., Erfani, H., Clark, S.L., Salmanian, B., Baker, B.W., et al. (2017) Multidisciplinary Team Learning in the Management of the Morbidly 
Adherent Placenta: Outcome Improvements over Time. American Journal of $O b$ stetrics \& Gynecology, 216, 612.e1-5.

[5] Committee on Obstetric Practice (2017) Committee Opinion No. 713: Antenatal Corticosteroid Therapy for Fetal Maturation. Obstetrics \& Gynecology, 130, e102e109. https://doi.org/10.1097/AOG.0000000000002237

[6] Wu, S., Kocherginsky, M. and Hibbard, J.U. (2005) Abnormal Placentation: Twenty-Year Analysis. American Journal of Obstetrics \& Gynecology, 192, 1458-1461. https://doi.org/10.1016/j.ajog.2004.12.074

[7] Maguire, P. (1998) Breaking Bad News. European Journal of Surgical Oncology, 24, 188-191. https://doi.org/10.1016/S0748-7983(98)92929-8 